\title{
ANÁLISE SENSORIAL DE DIFERENTES MARCAS COMERCIAIS DE CAFÉ (Coffea arabica L.) ORGÂNICO
}

\author{
Sensory evaluation of differents comercial marks of the organic coffee (Coffea arabica 1.$)$
}

\author{
Aline Fonseca da Silva ${ }^{1}$, Valéria Paula Rodrigues Minim² ${ }^{2}$ Milene Moreira Ribeiro ${ }^{3}$
}

\begin{abstract}
RESUMO
Produtos orgânicos são polêmicos quanto a sua qualidade sensorial. Neste estudo foi realizado um teste de aceitação em relação a um conjunto de atributos: cor, aroma, sabor e impressão global, no qual sessenta e seis consumidores avaliaram quatro amostras de café orgânico e uma amostra de café convencional de quatro diferentes marcas comerciais. Para a realização desse teste utilizou-se uma escala hedônica de nove pontos e os resultados obtidos foram avaliados por meio de Mapa de Preferência Interno (MDPREF). O MDPREF demonstrou que a amostra de café orgânico ORG-4 obteve maior aceitação pelos consumidores em relação aos quatro atributos avaliados, mostrando que nessa marca de café os atributos em conjunto torna-a bem mais apreciada. A marca de café orgânico ORG-1 foi menos preferida quanto aos atributos cor, sabor e impressão global. A amostra de café CON foi menos aceita quanto ao atributo aroma.
\end{abstract}

Termos para indexação: Café orgânico, qualidade, análise sensorial, Mapa de Preferência Interno.

\begin{abstract}
Organic products are controversial in relation to sensory quality. In that study an acceptance test was accomplished in relation to a group of attributes: color, aroma, flavor and overall impression, where sixty six consumers evaluated four samples of organic coffee and a sample of conventional coffee of four different commercial marks. For the accomplishment of that test a hedonic scale of nine points was used and the obtained results were analyzed by Internal Preference Map (PREFMD). PREFMD demonstrated that the sample of organic coffee ORG-4 obtained larger acceptance for the consumers in relation to the four attributes, showing that in that mark of coffee the attributes together turn it well appreciated. The mark of organic coffee ORG-1 was less accepted by the consumers in relation to the attributes color, flavor and overall impression. For the attribute aroma, the coffee CON was the one of smaller preference.
\end{abstract}

Index terms: organic coffee, quality, sensory evaluation, Internal Preference Map.

(Recebido para publicação em 22 de abril de 2004 e aprovado em 29 de agosto de 2005)

\section{INTRODUÇÃO}

O café é um produto consumido diariamente no mundo por todas as classes sociais. É uma cultura com grande incidência de pragas e doenças fazendo com que a utilização de produtos químicos, seja em larga escala. Atualmente, a população mundial vem se preocupando com questões ambientais e com os prejuízos causados a saúde devido ao uso indiscriminado de agrotóxicos e por isso estão preferindo produtos mais saudáveis.

No Brasil, a principal motivação para a aquisição de alimentos orgânicos também está ligada à preocupação com a saúde. Uma pesquisa realizada com consumidores da região Sul e Sudeste do Brasil pelo Datacenso (2002) e apresentada por Darolt (2003), mostrou que os principais motivos para o consumo de alimentos orgânicos foram: $1^{\circ} \mathrm{e}$ $2^{\circ}$ lugares- faz bem à saúde; $3^{\circ}$ lugar- não tem agrotóxicos; $4^{\circ}$ lugar- mais sabor, e em $5^{\circ}$ lugar- mais natural e com mais qualidade. E ainda concluiu que, quem consome os orgânicos são adultos e idosos pertencentes às classes $\mathrm{A}$ e $\mathrm{B}$.

Em trabalho desenvolvido no Reino Unido, Brennan \& Kuri (2002) mostraram que além do preço o produto orgânico tem sua aceitação influenciada pelos atributos sensoriais.

Os sistemas orgânicos de produção de café criaram um nicho de mercado muito peculiar de cafés especiais, o dos cafés orgânicos. Esse segmento de mercado é o que mais cresce no mundo, chegando, nos últimos cinco anos, a uma taxa de $500 \%$ ao ano. O marketing relacionado com produtos orgânicos é feito por todos os grupos ambientalistas do mundo. A produção brasileira de café orgânico ainda é relativamente limitada e é basicamente direcionada ao mercado externo (LEITE \& SILVA, 2000).

As indústrias de alimentos têm buscado identificar e atender os anseios dos consumidores em relação a seus produtos, pois só assim sobreviverão num mercado cada

${ }^{1}$ Engenheira Agrônoma, Msc. - Departamento de Ciência e Tecnologia de Alimentos/UFV - alinefonseca3@yahoo.com.br

²Engenheira de Alimentos, D.S., Professora Adjunto IV - Departamento de Ciência e Tecnologia de Alimentos/UFV - vprm@ufv.br

${ }^{3}$ Estudante de graduação de Engenharia de Alimentos - Departamento de Ciência e Tecnologia de Alimentos/UFV - milene.ribeiro@bol.com.br 
vez mais competitivo (CARNEIRO, 2001). A determinação da aceitação pelo consumidor é parte crucial no processo de desenvolvimento ou melhoramento de produtos. Os testes afetivos requerem equipe com grande número de participantes e que representem a população de consumidores atuais e/ou potenciais do produto. Entre os métodos mais empregados na medida de aceitação de produtos está a escala hedônica, em que o consumidor expressa sua aceitação pelo produto seguindo uma escala previamente estabelecida que varia gradativamente com base nos termos "gosta" e "desgosta" (CHAVES \& SPROSSER, 2001).

Os resultados de testes afetivos, tradicionalmente são avaliados por ANOVA e testes de comparação de médias, comparando-se a aceitação média entre produtos. Segundo Polignano et al. (1999), esta análise global considerando conjuntamente as avaliações de todos os consumidores, implica em assumir que todos apresentam o mesmo comportamento, desconsiderando suas individualidades e que os dados podem não estar sendo bem visualizados a ponto de se perder informações interessantes sobre diferentes segmentos de mercado. A técnica de Mapa de Preferência pode solucionar este problema e também permitir a associação da impressão que os consumidores têm de um produto, com suas características sensoriais.

Há uma carência de informações voltadas para a análise sensorial dos produtos orgânicos, principalmente produtos orgânicos processados. Alguns autores acreditam que os alimentos orgânicos sejam de qualidade sensorial superior aos produzidos de modo convencional.

De acordo com Darolt (2003), os estudos comparativos que focam a qualidade sensorial estão em estágio inicial e mostram resultados variáveis, o que não permite afirmar que existem diferenças estatísticas entre sabor e aroma de produtos orgânicos ou convencionais.

Com presente trabalho, objetivou-se avaliar a aceitação de café (Coffea arabica L.) orgânico torrado e moído encontrado no mercado brasileiro.

\section{MATERIAL E MÉTODOS}

\section{Amostras}

Foram avaliadas quatro amostras de café orgânico torrado e moído (ORG-1, ORG-2, ORG-3, ORG-4) e uma amostra de café convencional torrado e moído (CON) proveniente de diferentes marcas comerciais. Os cafés ORG-4 e CON são de mesma marca. As amostras utilizadas nesse trabalho são provenientes do mesmo tipo de torra e lote de fabricação.

\section{Teste de Aceitação}

As amostras foram avaliadas por 66 consumidores, sendo 23 mulheres e 43 homens, com faixa etária entre 16 e 44 anos.

O teste de aceitação foi realizado no Laboratório de Análise Sensorial do Departamento de Tecnologia de Alimentos da Universidade Federal de Viçosa-MG, em novembro de 2002.

De acordo com a recomendação de Carvalho et al. (1997), as amostras foram preparadas com água deionizada e pó de café na proporção de $6 \%$, ou seja, para cada $30 \mathrm{~g}$ de pó de café foi utilizado $500 \mathrm{~mL}$ de água deionizada. A bebida foi preparada em uma cafeteira elétrica Wallita Therm RI6492, coado em papel filtro $\mathrm{n}^{\circ} 102$ da marca Jovita e armazenado em garrafa térmica da marca Alladin no máximo por uma hora para que a bebida não fosse servida em temperatura inadequada.

As amostras foram servidas de forma monádica aos consumidores em cabines individuais. Para avaliação de aroma, sabor e impressão global as xícaras foram cobertas por vidro de relógio. $\mathrm{O}$ atributo cor foi avaliado fora da cabine com o uso da luz natural em béquer de $250 \mathrm{~mL}$. Junto a cada amostra servida, o consumidor recebeu um copo de água em temperatura ambiente para enxaguar a boca entre as avaliações. Foram servidos aos provadores sachês de açúcar e adoçante para que pudessem adoçar o café de acordo com a preferência. O consumidor recebeu uma ficha resposta, na qual pedia-se a ele que marcasse o seu julgamento em relação à aceitação do café (Quadro 1).

\section{Análise dos resultados}

Os resultados obtidos no teste de aceitação foram avaliados por meio da técnica de Mapa de Preferência Interno. Para a obtenção do MDPREF os dados de aceitação foram organizados em uma matriz de amostras (linhas) e consumidores (colunas) e esta submetida à Análise de Componentes Principais (ACP) (CARNEIRO, 2001). Os resultados foram expressos em um gráfico em que as amostras estão representadas por figuras e os consumidores são representados por pontos.

As análises estatísticas foram realizadas utilizando os procedimentos do programa estatístico SAS Institute (1999) (Statistical Analysis System-SAS Institute Inc. North Carolina, USA), versão 8.0 licenciado para Universidade Federal de Viçosa. 
QUADRO 1 - Modelo de ficha para o teste de aceitação do café.

\begin{tabular}{|lll|}
\hline & \multicolumn{3}{c}{ TESTE DE ACEITAÇÃO } & \\
Nome: & & \\
Por favor, avalie a amostra codificada e use a escala abaixo para indicar o quanto você gostou ou desgostou da \\
amostra. & & \\
Código da amostra: & Impressão global & \\
9- gostei extremamente & Aroma & \\
8- gostei muito & Sabor & \\
7- gostei moderadamente & Cor & \\
6- gostei ligeiramente & & \\
5- nem gostei / nem desgostei & & \\
4- desgostei ligeiramente & & \\
3- desgostei moderadamente & & \\
2- desgostei muito & & \\
1-desgostei extremamente & & \\
\hline
\end{tabular}

\section{Análise dos resultados}

Os resultados obtidos no teste de aceitação foram avaliados por meio da técnica de Mapa de Preferência Interno. Para a obtenção do MDPREF os dados de aceitação foram organizados em uma matriz de amostras (linhas) e consumidores (colunas) e esta submetida à Análise de Componentes Principais (ACP) (CARNEIRO, 2001). Os resultados foram expressos em um gráfico em que as amostras estão representadas por figuras e os consumidores são representados por pontos.

As análises estatísticas foram realizadas utilizando os procedimentos do programa estatístico SAS Institute (1999) (Statistical Analysis System-SAS Institute Inc. North Carolina, USA), versão 8.0 licenciado para Universidade Federal de Viçosa.

\section{RESULTADOS E DISCUSSÃO}

Mapa de preferência interno de aceitação quanto à cor

O Mapa de Preferência Interno foi efetuado utilizando os dados obtidos no teste de aceitação quanto à cor das cinco amostras de café. Os dois componentes principais explicam $70 \%$ da variação da aceitação das amostras de café em relação à cor, o primeiro componente principal e o segundo componente principal explicam $41 \%$ e $29 \%$, respectivamente.

Na Figura 1, observa-se a dispersão das amostras e a correlação dos dados de aceitação com os dois componentes principais. Nessa representação gráfica as amostras são representadas por símbolos e os consumidores estão representados por pontos. A separação espacial das amostras mostra a formação de quatro grupos separados por marcas, indicando a diferença na aceitação em relação à cor. Um grupo composto pelas amostras de café ORG-4 e ORG-3; um segundo grupo pela amostra CON, o terceiro grupo pela amostra ORG-1 e o quarto grupo pela amostra ORG-2. Os consumidores estão localizados próximo à região das amostras que eles mais preferiram. Diante disso, as amostras ORG-4, ORG-3 e a CON foram as preferidas, pois a maior parte dos consumidores deram notas altas para essas amostras e amostra ORG-1 apresentou-se como a de menor preferência pelos consumidores quanto a cor. Os consumidores que estão alocados na região central do gráfico não estão correlacionados com os dois componentes, conseqüentemente contribuíram pouco para discriminar as amostras em relação à cor, para esses consumidores todas as amostras tiveram a mesma preferência. 


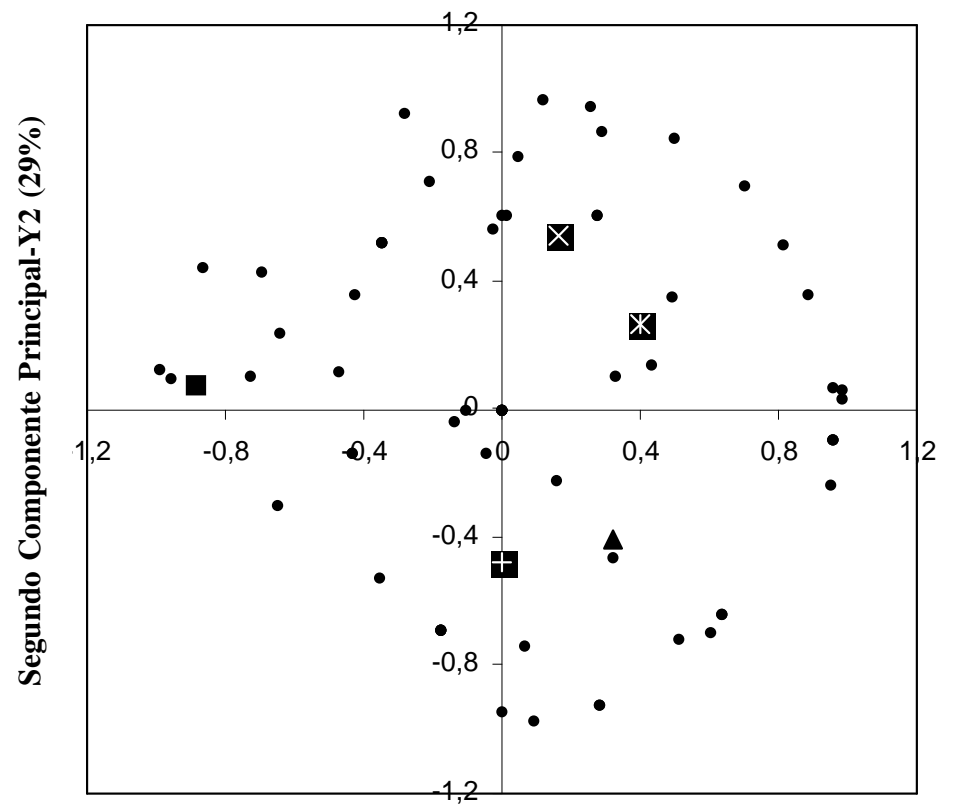

Primeiro Componente Principal-Y1 (41\%)

FIGURA 1 - Mapa de Preferência Interno para as amostras de café orgânico e convencional em relação à cor.

\section{Mapa de preferência interno de aceitação quanto ao sabor}

O Mapa de Preferência Interno foi gerado por dois componentes que explicaram juntos $63 \%$ da variação existente entre as amostras de café em relação ao sabor. $\mathrm{O}$ primeiro componente principal explica $35 \%$ da variação enquanto o segundo componente explica $28 \%$ da variação entre as amostras de café. Na Figura 2, observa-se que as marcas ORG-3, ORG-4 são semelhantes entre si, sendo estas as mais preferidas pelos consumidores, diferindo das marcas CON, ORG-2 e ORG-1, sendo esta última de menor aceitação quanto ao sabor.

\section{Mapa de preferência interno de aceitação quanto ao aroma}

De acordo com os dados obtidos no teste de aceitação de cinco amostras distintas de café torrado e moído foi realizado o Mapa de Preferência Interno para aroma .

O primeiro componente principal explica 39\% da variação existente entre as amostras de café quanto ao aroma e o segundo componente principal explica $29 \%$ da variação. Os dois primeiros componentes principais explicam juntos $68 \%$ da variação entre as amostras em relação ao atributo aroma. Na Figura 3, nota-se que a separação espacial das amostras para o atributo aroma tiveram o mesmo comportamento quando comparado ao atributo sabor e cor, ocorrendo formação de quatro grupos distintos. As amostras ORG-3e ORG-4 apresentam-se como as mais aceitas pelos os consumidores e a amostra CON como a amostra de menor aceitação.

\section{Mapa de preferência interno de aceitação quanto à impressão global}

As amostras foram avaliadas pelo conjunto de atributos: aroma, sabor e aparência. O Mapa de Preferência Interno gerou um espaço multidimensional, que foi gerado por meio de componentes principais, que juntos explicaram $66 \%$ da variação existente entre as amostras de café em relação à impressão global. $\mathrm{O}$ primeiro componente principal explicou $42 \%$ da variação existente entre as amostras e o segundo componente principal explicou $24 \%$.

Pela Figura 4, observa-se que há formação de quatro grupos distintos em relação à impressão global, sendo o grupo 1 composto pela amostra ORG-4, o grupo 2 pelas amostras ORG-3 e CON, o grupo 3 pela amostra ORG-1 e o quarto grupo formado pela amostra ORG-2.

A amostra de café ORG-4 demonstrou ser a amostra de maior aceitação pelos consumidores quanto à impressão global, mostrando que nessa marca de café os atributos em conjunto torna-a bem apreciada e de maior aceitação, sendo a amostra ORG-1 de menor preferência em relação à impressão global. 


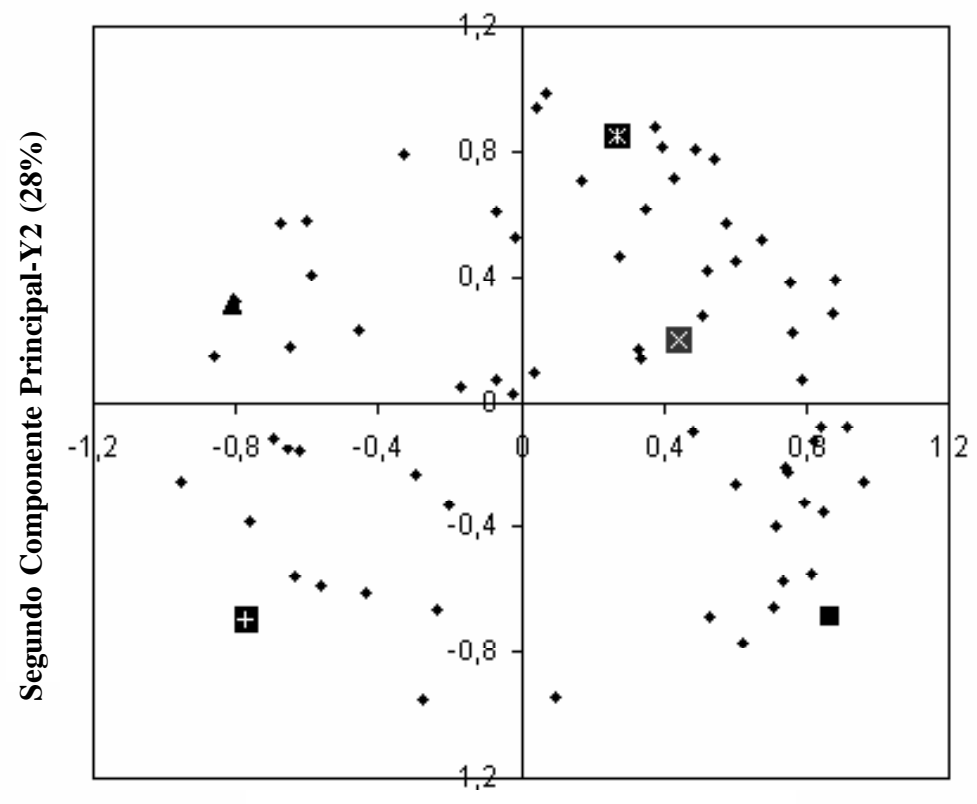

Primeiro Componente Principal-Y1 (35\%)

FIGURA 2 - Mapa de Preferência Interno para as amostras de café orgânico e convencional em relação ao sabor.

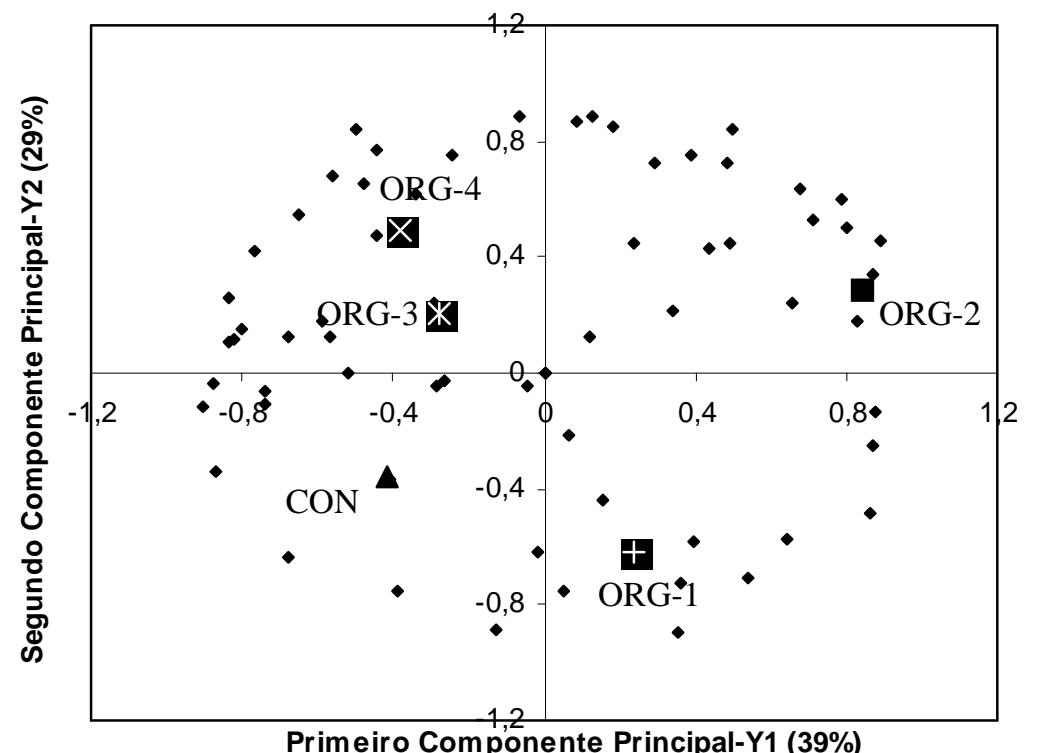

FIGURA 3 - Mapa de Preferência Interno para as amostras de café orgânico e convencional em relação ao aroma. 


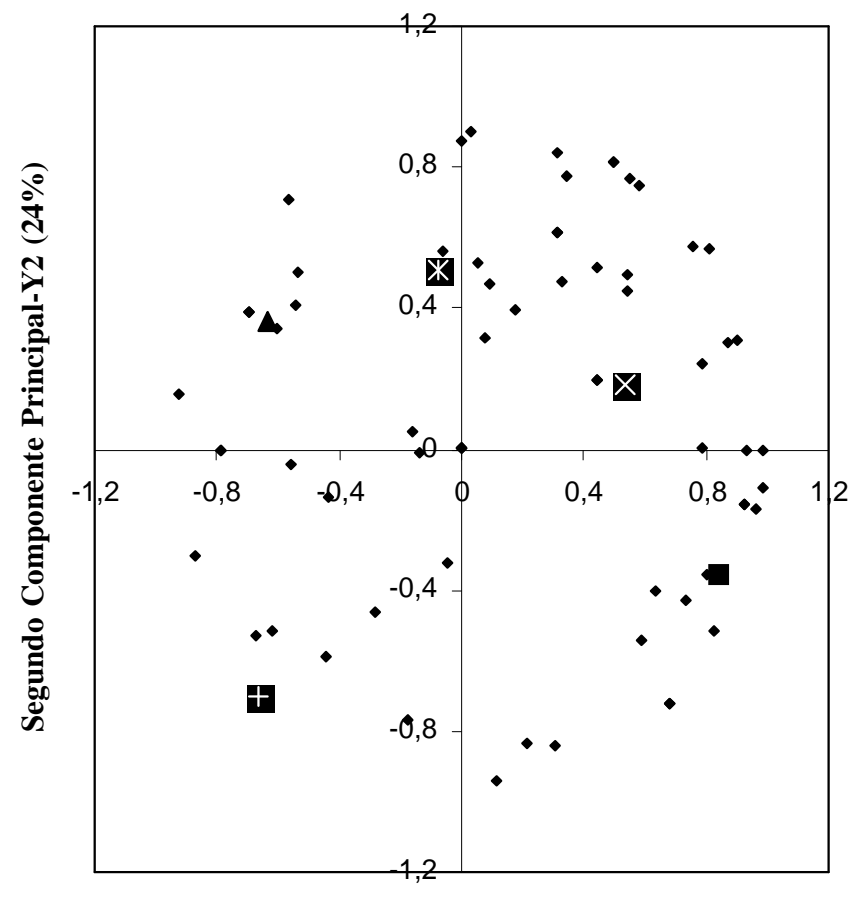

Primeiro Componente Principal-Y1 (42\%)

FIGURA 4 - Mapa de Preferência Interno para as amostras de café orgânico e convencional em relação à impressão global.

\section{CONCLUSÃO}

De acordo com o teste de aceitação, as marcas ORG-4 e ORG-3 foram mais preferidas quanto aos atributos cor, sabor e aroma. Em relação ao atributo impressão global a amostra mais aceita foi a de café ORG-4. A marca de café orgânico ORG-1 foi menos preferida pelos consumidores quanto aos atributos cor, sabor e impressão global. Para o atributo aroma a marca de café $\mathrm{CON}$ foi a de menor preferência.

Entre as marcas de café orgânico avaliadas houve diferença na aceitação.

Por serem marcas diferentes o processamento dado pelas indústrias pode ter sido diferenciado ou pelo fato da aquisição de matéria-prima ser de diferentes produtores e estes não tenham prezado pela qualidade do grão conferiu a uma bebida de qualidade inferior, podendo ter sido fator determinante para a aceitação.

Em relação às amostras ORG-4 e CON provenientes de uma mesma marca observou-se que a amostra de café orgânico foi mais aceita pelos consumidores em relação a convencional.

\section{REFERÊNCIAS BIBLIOGRÁFICAS}

BRENNAN, C. S.; KURI, V. Relationship between sensory attributes, hidden attributes and price in influencing consumer perception of organic foods. In: CONFERENCE UK ORGANIC RESEARCH, 2002, Aberystwyth. Proceedings... Aberystwyth: [s.n.], 2002.

CARNEIRO, J. C. S. Processamento industrial de feijão e avaliação sensorial, descritiva e mapa de preferência. 2001. 90 f. Dissertação (Mestrado em Ciência e Tecnologia de Alimentos) - Universidade Federal de Viçosa, Viçosa, 2001.

CARVALHO, V. D. de; CHAGAS, S. J. de R.; CHALFOUN, S. M. Fatores que afetam a qualidade do café. Informe Agropecuário, Belo Horizonte, v. 18, n. 187, p. 5-20, 1997.

CHAVES, J. B. P.; SPROSSER, R. L. Práticas de laboratório de análise sensorial de alimentos e bebidas. Viçosa: UFV, 2001. $81 \mathrm{p}$. 
DAROLT, M. R. Comparação entre a qualidade do alimento orgânico e a do convencional. In: STRINGHETA, P. C.; MUNIZ, J. N. Alimentos orgânicos: produção, tecnologia e certificação. [S.1.: s.n.], 2003. p. 289-312.

DATACENSO. Mercado de produtos orgânicos: consumidor. Curitiba: SEBRAE, 2002. 89 p.

LEITE, C. A. M.; SILVA, O. M. A demanda de cafés especiais. In: Café: produtividade, qualidade e sustentabilidade. Viçosa: UFV, 2000. p. 51- 73.
POLIGNANO, L. A. C.; CHENG, L. C.; DRUMOND, F. B. Utilização dos mapas de preferência como técnicas auxiliares do QFD durante o desenvolvimento de produtos alimentícios. In: CONGRESSO BRASILEIRO DE GESTÃO DO DESENVOLVIMENTO DE PRODUTO, 2., 1999, Belo Horizonte. Anais... Belo Horizonte: [s.n.], 1999. p. 274-294.

SAS INSTITUTE. SAS software version 8. Cary, 1999. 